\title{
EXPERIMENTAl SET-UP of a PUREly Electrostatic Monochromator for High Resolution and analytical Purposes of a 200 KV TEM
}

\author{
Stephan Uhlemann \& Max. Haider
}

CEOS GmbH, Englerstr. 28, D-69126 Heidelberg, Germany

Modern transmission electron microscopes equipped with field emitters have an information limit which exceeds the point resolution - given by the wave length and the spherical aberration constant $\mathrm{C}_{3}$ at Scherzer defocus. With Cs-correctors the point resolution is improved up to the information limit. Therefore, the spherical aberration corrected TEM suffers mainly from chromatic aberration $\mathrm{Cc}$ and the primary energy width $\Delta \mathrm{E}$. The chromatic aberration constant $\mathrm{Cc}$, however, cannot as easily be compensated as the spherical aberration. Therefore, instead of reducing Cc we have started a project to reduce the energy width of the illumination system for such a modern instrument.

The reduction of the energy width is not only advantageous for Cs-corrected TEMs but also for imaging techniques with numerical correction of spherical aberration- such as electron holography and focus variation - and, very important, for electron energy loss spectroscopy (EELS) with high energy resolution. For the latter application a small energy width even down to about $100 \mathrm{meV}$ at large probe currents has to be achieved.

The concept we are following has been proposed by Kahl and Rose [1]. It is a purely electrostatic $\Omega$-shaped energy filter with the energy selecting slit in the symmetry plane of this filter (s. Fig.1a). The image of the electron source in the dispersive plane is a line focus, a few $10 \mu \mathrm{m}$ in height. The energy selecting slits rang from 1 to $7 \mu \mathrm{m}$. The dispersion cancels completely after the monochromator. The load-free bipolar voltage supply provides a superior reproducibility of beam within the monochromator. Moreover it allows a fast and hysteresis free switch to turn it on and off.

The monochromator is incorporated in a UHV chamber above the accelerator and is driven at extractor potential of the Schottky-emitter. With a gun lens and its movable aperture the beam can be focused into the energy dispersive plane. The selectable gun-lens aperture allows the selection of the emission angle of the electron source, hence, the beam current. The energy selecting slit system consists of four selectable slits with a width of $1 \mu \mathrm{m}, 3 \mu \mathrm{m}, 5 \mu \mathrm{m}$ and $7 \mu \mathrm{m}$. The dispersion of the monochromator depends linearly on the extractor potential, which defines the energy of the electrons within the monochromator. At $4 \mathrm{kV}$ extractor voltage the dispersion is $12.0 \mu \mathrm{m} / \mathrm{eV}$ and, therefore, with the given width of the slit at this extractor setting the theoretical limit of the smallest energy width one might achieve is about $80 \mathrm{meV}$ FWHM.

The monochromator has been constructed and incorporated into the accelerator UHV chamber. In a first step, the electron probe has been analysed without acceleration in a low voltage SEM. (an old Philips 525 SEM). For the measurement of the energy width a faraday cage has been designed with an electrically isolated grid in front of it. If we apply a voltage to this grid the faraday cup can act as an edge spectrometer. The voltage is linearly ramped up from $5 \mathrm{~V}$ below to $5 \mathrm{~V}$ above the extractor voltage and the current at the faraday cage then is differentiated, which gives the distribution of the electrons over this energy range. For improving the signal to noise ratio 256 records are accumulated. The energy resolution of this spectrometer has been measured to be $40 \mathrm{meV}$. The first results obtained with this monochromator are shown in Fig. 2. The measured energy width of the filtered beam is $\Delta \mathrm{E}=90 \mathrm{meV} F W H M$, still having a beam current of $1 \mathrm{nA}$. The aperture at the tippotential is $5 \mathrm{mrad}$ in this case. The excitation of the emitter yields an angular intensity of $115 \mu \mathrm{A} / \mathrm{sr}$. When using a smaller aperture $(3 \mathrm{mrad})$ the filtered beam current was reduced to $0.36 \mathrm{nA}$ and the energy width was measured to be $\Delta \mathrm{E}=81 \mathrm{meV}$ FWHM. This demonstrates a very good 
agreement with the specifications in the German SESAMe project. Which assumes $200 \mathrm{meV}$ for the filtered beam before entering the accelerator. This development has been carried out in collaboration with LEO/Germany for their new generation of advanced TEMs.

References:

[1] F. Kahl F. \& H. Rose, Proc. EUREM Brno/Cz, Vol.III (2000) 1459

a)

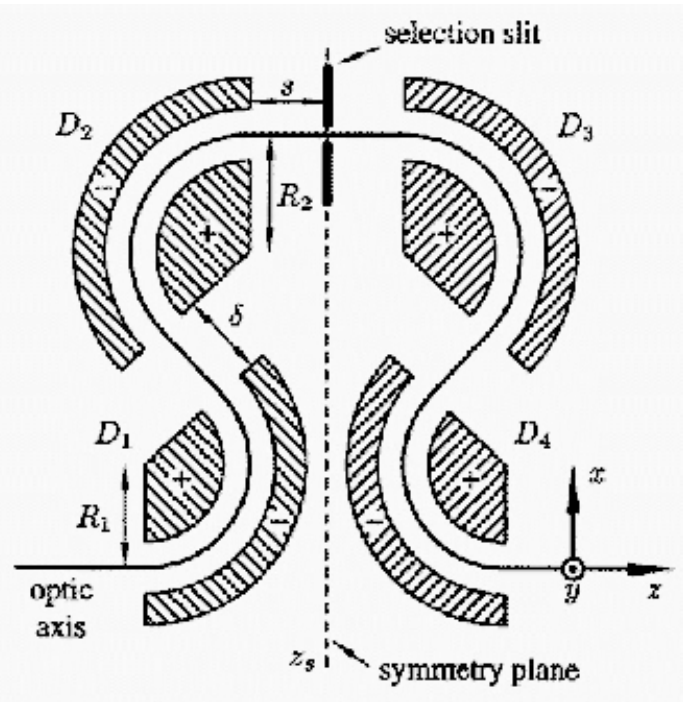

b) 2 cin

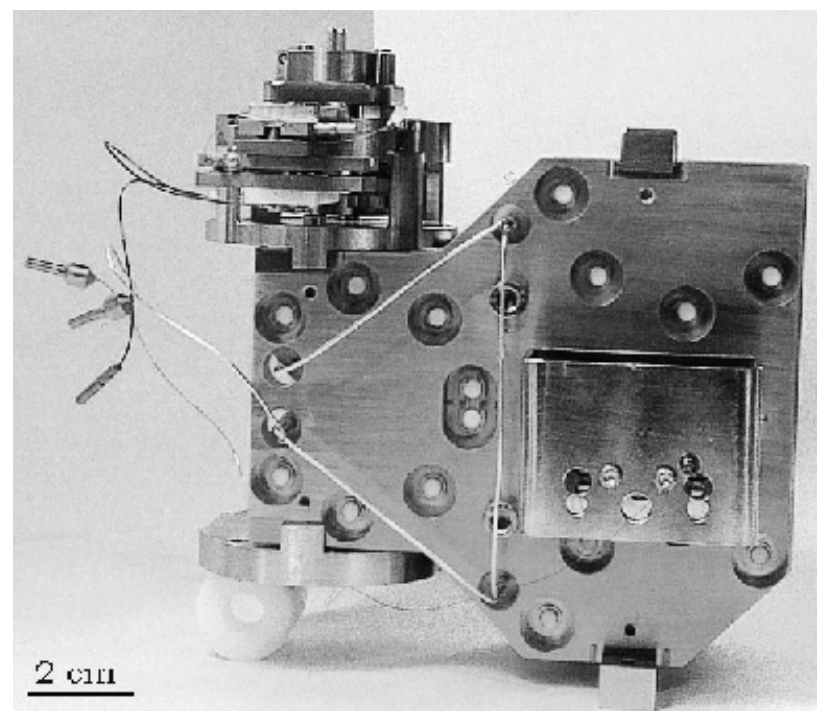

Fig.:1 a) Schematic drawing of the monochromator and b) the monochromator, turned by 90 to the right compared with the drawing, after finishing the construction.

Aperture 5mrad, Ex 4kV, Tf 1744K, 115uA/sr (20.12.01)

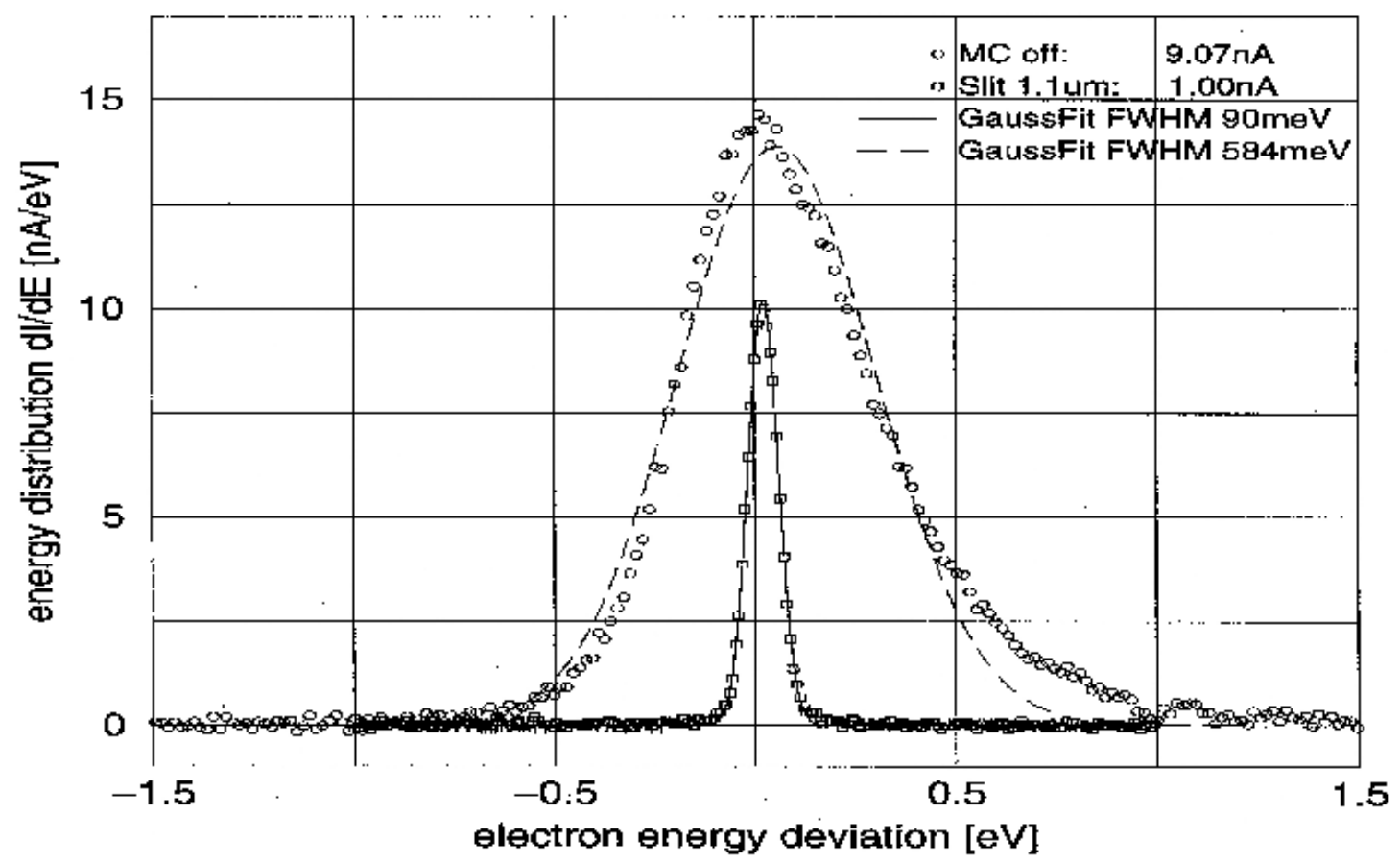

Fig.:2 Measured energy distributions of the Schottky emitter without (circles) and with filtering (squares). The energy slit was $1.0 \mu \mathrm{m}$ and the dispersion $12 \mu \mathrm{m} / \mathrm{eV}$. 